\title{
Nonlocalized Generation of Correlated Photon Pairs in Degenerate Down-Conversion
}

\author{
Kayn A. Forbes, Jack S. Ford, and David L. Andrews \\ School of Chemistry, University of East Anglia, Norwich NR4 7TJ, United Kingdom
}

(Received 30 November 2016; published 30 March 2017)

\begin{abstract}
The achievement of optimum conversion efficiency in conventional spontaneous parametric downconversion requires consideration of quantum processes that entail multisite electrodynamic coupling, actively taking place within the conversion material. The physical mechanism, which operates through virtual photon propagation, provides for photon pairs to be emitted from spatially separated sites of photon interaction; occasionally pairs are produced in which each photon emerges from a different point in space. The extent of such nonlocalized generation is influenced by individual variations in both distance and phase correlation. Mathematical analysis of the global contributions from this mechanism provides a quantitative measure for a degree of positional uncertainty in the origin of down-converted emission.
\end{abstract}

DOI: 10.1103/PhysRevLett.118.133602

Spontaneous parametric down-conversion (SPDC) is a process in which light passing through an optically nonlinear medium can generate double-wavelength output. At a fundamental level, the process entails the conversion of pump photons into conascent, phase-matched pairs, executed by material interactions that entail the secondorder nonlinear optical susceptibility: a third-order electric dipole response. Each generated pair of photons has a combined energy and momentum equal to that of the corresponding annihilated photon, and they also exhibit correlated polarization. When the emergent photons equally share the energy of the input, the process is known as degenerate down-conversion (DDC); an alternative perspective is to regard the conversion as an exact time reversal of second-harmonic generation [1,2]. Much work has been carried out on correlated photon pairs, including their generation [3-5], manipulation [6-9], and application [10-12]. The entanglement of the quantum states in each pair has important applications in quantum computing and communication [13], and potential utilization clinically [14].

In this Letter we develop an expanded theory of SPDC, highlighting and quantifying an important contribution from nonlocalized couplings. While SPDC is one of the main sources of entangled photon pairs [15], an exact location for the creation of each output photon cannot of course be inferred by direct observation-although pump photon annihilation and down-converted photon emission are generally assumed to be colocated. Of course, the diffuse nature of atomic and molecular orbitals precludes exact identification of the location for any photon creation event; equally, even the emission of two correlated photons cannot be considered as precisely colocated in origin. However, the spatial extent of the region from within which a pair of down-converted photons may emerge is considerably larger than may usually be supposed.

In fact, there is a possibility that for each input photon the process may entail correlated photon interactions at two separate locations, creating one down-converted photon at each as indicated in Fig. 1. Accounting for such delocalized interactions provides fresh insights into the extent of nonlocalization in the origin of photon pairs. In principle, this effect enables a pair of correlated photons to emerge from two spatially separated origin points, and this introduces new positional uncertainty of a fundamental quantum origin. In the following analysis, using a quantum electrodynamical (QED) formalism, we fully account for both localized and nonlocalized emission. The results are then developed for computational implementation, aiming to quantify the net effect of nonlocalization within a model lattice structure. It is thus shown that multicenter components contribute to the overall rate of down-converted frequency generation, introducing positional uncertainty of a fundamental quantum origin.

The primary analysis is carried out within a nonrelativistic quantum electrodynamical formulation [16-18], framed on the Power-Zienau-Woolley Hamiltonian [19]. The multipolar formalism [20] is specifically adopted to describe the interactions of matter and radiation within the electromagnetic field. In this theory, all events that lead to observable phenomena explicitly occur through material-based annihilation and creation of photons, and all nonlocal couplings are mediated by fluctuations in the vacuum field, i.e., through virtual photon propagation [21]. Bulk media effects are systematically accounted for by the incorporation of the appropriate refractive and dissipative factors [22].

We first designate as point $A$ the location for the annihilation of any single input photon, and the creation of at least one output photon. To ensure local energy conservation over a time scale beyond the conversion event, any separate location (labeled $B$ ) that is the creation site for the other photon must be coupled to $A$ by virtual photon exchange, as indicated by the double-headed arrow in Fig. 1. (The possibility of neither down-converted photon emerging from the site of input photon annihilation 

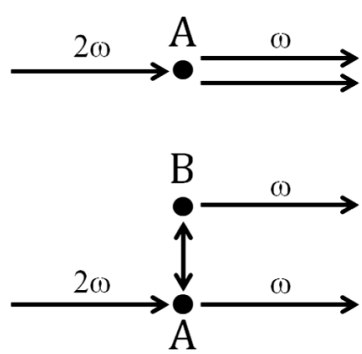

FIG. 1. Schematic for two SPDC mechanisms. Above: Photon annihilation and both photon creation events are colocated at $A$. Below: The annihilation and one creation occur at $A$, the other creation event at another location $B$. Points $A$ and $B$ are coupled via virtual photon exchange, indicated by the double-headed arrow. In the full calculation, all time orderings are taken into account, and the positional geometry is arbitrary.

represents a much weaker, higher-order interaction to be discussed in subsequent work.) Using the standard framework of time-dependent perturbation theory and the Fermi rule [23] for the system as a whole, the observable rate of down-conversion is expressible as

$$
\Gamma_{\mathrm{SPDC}}=2 \pi \hbar^{-1} \rho\left|\sum_{A} M_{A}+\sum_{A, B} M_{A B}\right|^{2},
$$

where $\rho$ is the density of final states of the radiation. For the conventional single-center mechanism of parametric down-conversion the use of third-order time-dependent perturbation theory delivers the standard result for the matrix element $M_{A}$ involving three photon interactions. Explicitly calculating the other, more complicated $M_{A B}$ elements in (1) requires a development using fifth-order perturbation theory, due to the additional virtual photon creation and annihilation events. To this end, and to circumvent arduous evaluation of all the time orderings based on conventional Feynman graphs, we deploy the more expedient state-sequence methodology [24,25].

For coherent down-conversion through the single-site mechanism, the photon momentum of the radiation field is conserved, and therefore the net output is matched in phase to the input. To effect a degree of simplification that in no way affects the impact of the analysis to follow, we assume index-matching, which implies forward emission. Using textbook methods of QED [18], including refractive media corrections [22], then by invoking the Einstein rule for summation over repeated indices, the matrix element $M_{A}$ is cast as

$M_{A}=\sum_{A} \frac{i}{4}\left(\frac{\hbar \omega}{n}\right)^{\frac{3}{2}}\left(\frac{q}{\varepsilon_{0} V}\right)^{\frac{1}{2}} e_{i} \bar{e}_{j}^{\prime} \bar{e}_{k}^{\prime} \chi_{i(j k)}^{(2)}(-\omega,-\omega ; 2 \omega)$,

where $\chi_{i(j k)}^{(2)}(-\omega,-\omega ; 2 \omega)$ is the second-order susceptibility [1], $2 \omega$ is the optical frequency for the pump beam of wave vector $\boldsymbol{k}$, and $\omega$ is that of the output mode $\boldsymbol{k}^{\prime} ; n$ is the complex refractive index; $\boldsymbol{e}$ and $\boldsymbol{e}^{\prime}$ are the polarization vectors of the incident and emitted photons, respectively. The quantity $q / V$ quantifies the irradiance of the pump beam: $q$ is the mean occupation number for the state of the incident radiation mode within a volume $V$, the latter signifying the effective volume of each distinct active center. The product polarization tensor $e_{i} \bar{e}_{j}^{\prime} \bar{e}_{k}^{\prime}$ is clearly $j, k$ symmetric, and thus only the $j, k$-symmetric part of the nonlinear susceptibility tensor $\chi_{i j k}^{(2)}$, with which it contracts, can contribute: written as $\chi_{i(j k)}^{(2)}$ the explicit formula for the index-symmetric form is conventionally cast as follows:

$$
\begin{aligned}
& \chi_{i(j k)}^{(2)}(-\omega,-\omega ; 2 \omega) \\
& =\frac{N}{2 \varepsilon_{0}}\left(\frac{n^{2}+2}{3}\right)^{3} \sum_{r, s}\left[\left(\frac{\mu_{i}^{0 r} \mu_{j}^{r s} \mu_{k}^{s 0}}{\left(E_{s 0}-\hbar \omega\right)\left(E_{r 0}-2 \hbar \omega\right)}\right.\right. \\
& \left.\quad+\frac{\mu_{j}^{0 r} \mu_{i}^{r s} \mu_{k}^{s 0}}{\left(E_{s 0}-\hbar \omega\right)\left(E_{r 0}+\hbar \omega\right)}+\frac{\mu_{j}^{0 r} \mu_{k}^{r s} \mu_{i}^{s 0}}{\left(E_{s 0}+2 \hbar \omega\right)\left(E_{r 0}+\hbar \omega\right)}\right) \\
& \quad+\{j \leftrightarrow k\}],
\end{aligned}
$$

where $N$ is the number density of active centers, equivalent to $V^{-1}$, and $\boldsymbol{\mu}^{a b}$ is a generic electric transition dipole moment for the $a \leftarrow b$ electronic transition; $\{j \leftrightarrow k\}$ signifies terms obtained by exchanging these indices.

The next step in calculating the overall rate (1) is to calculate the second term in the modulus square. To relate

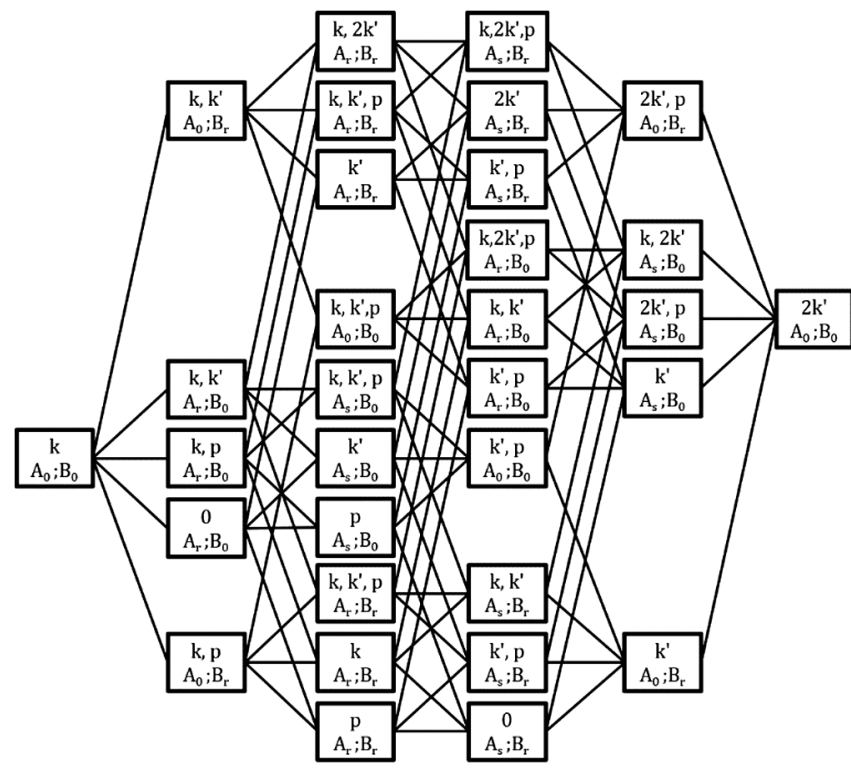

FIG. 2. State sequence diagram accommodating 120 path contributions for two-center down-conversion, with the initial state of the radiation field and matter on the far left and the corresponding final state on the right; intervening entries represent intermediate states; $k$ and $k^{\prime}$ denote input and output mode photons, $p$ a virtual photon. Subscripts on $A$ and $B$ denote electronic states, 0 the ground state, and $r, s$ virtual states. 
photon events at disparate locations we define as $\boldsymbol{r}_{B}$ the vector displacement of each correlated interaction site $B$, from the position of $A$. Without prejudice in regard to time ordering, the overall process is thus represented as a photon of frequency $2 \omega$ being annihilated at $A$, with coupling between $A$ and $B$ via virtual photon propagation leading to the emission of a photon of frequency $\omega$ from $B$, and a counterpart photon of the same frequency also emerges from $A$. Overall, the electronic state of the material is both locally and globally unchanged, unlike that of the radiation field.
The use of a state-sequence diagrammatic approach facilitates accounting for all of the 5 ! time orderings that contribute to the fifth-order matrix element. The structure of the diagram-which assimilates information from all 120 corresponding Feynman diagrams-is shown in Fig. 2. To exemplify one such matrix element contribution, the following is the result for the state sequence represented by the uppermost pathway in the figure, where sums are taken over virtual states $r, s$, and also over all wave vectors $\mathbf{p}$ and polarizations $\phi$ for the virtual photon,

$$
\begin{aligned}
M_{A B}= & \frac{i}{4 N} \sum_{r, s} \sum_{\boldsymbol{p}, \phi}\left(\frac{\hbar \omega}{n}\right)^{\frac{3}{2}}\left(\frac{\hbar c p}{\varepsilon_{0} V n}\right)\left(\frac{q \varepsilon_{0}}{V}\right)^{\frac{1}{2}}\left(\frac{n^{2}+2}{3}\right)^{5} e_{i} \bar{e}_{k}^{\prime} \bar{e}_{m}^{\prime} \bar{e}_{l}^{(\phi)} e_{j}^{(\phi)} \mu_{m}^{r 0(B)} \mu_{k}^{r 0(A)} \mu_{l}^{s r(A)} \mu_{i}^{0 s(A)} \mu_{j}^{0 r(B)} \\
& \times\left[\left(E_{0 r}^{B}-\hbar c k^{\prime}\right)\left(E_{0 r}^{A}+E_{0 r}^{B}-2 \hbar c k^{\prime}\right)\left(E_{0 s}^{A}+E_{0 r}^{B}-\hbar c\left(2 k^{\prime}+p\right)\right)\left(E_{0 r}^{B}-\hbar c p\right)\right]^{-1} e^{i n\left(\boldsymbol{p}-\boldsymbol{k}^{\prime}\right) \cdot \boldsymbol{r}_{B}}
\end{aligned}
$$

When all 120 such contributions are summed, the application of straightforward tensor algebra and calculus gives the overall quantum amplitude as

$$
\begin{aligned}
M_{A B}= & \sum_{A, B} \frac{i}{4 N}\left(\frac{\hbar \omega}{n}\right)^{\frac{3}{2}}\left(\frac{q \varepsilon_{0}}{V}\right)^{\frac{1}{2}} \frac{1}{n^{2}} \\
& \times e_{i} \bar{e}_{k}^{\prime} \bar{e}_{m}^{\prime} \chi_{i(j k)}^{(2)}(-\omega,-\omega ; 2 \omega) \chi_{l m}^{(1)}(-\omega ; \omega) \\
& \times V_{j l}\left(n k^{\prime}, \boldsymbol{r}_{B}\right) e^{-i n k^{\prime} \cdot r_{B}},
\end{aligned}
$$

where the second rank tensor $\chi_{l m}^{(1)}$ is the linear susceptibility, defined as

$$
\begin{aligned}
\chi_{l m}^{(1)}(-\omega, \omega)= & \frac{N}{\varepsilon_{0}}\left(\frac{n^{2}+2}{3}\right)^{2} \\
& \times \sum_{r}\left[\frac{\mu_{l}^{0 r(B)} \mu_{m}^{r 0(B)}}{E_{r 0}^{B}+\hbar \omega}+\frac{\mu_{m}^{0 r(B)} \mu_{l}^{r 0(B)}}{E_{r 0}^{B}-\hbar \omega}\right] .
\end{aligned}
$$

In calculating the quantum amplitude (5) the usual integrations over virtual photon wave vector and polarizations have been carried out, giving the term $V_{j l}\left(n k^{\prime}, \boldsymbol{r}_{B}\right)$, the retarded resonant dipole-dipole coupling tensor, as defined by

$$
\begin{aligned}
V_{i j}(n k, \boldsymbol{R})= & \frac{1}{4 \pi \varepsilon_{0} R^{3}}\left[\left(\delta_{i j}-3 \hat{R}_{i} \hat{R}_{j}\right)(1-i n k R)\right. \\
& \left.-\left(\delta_{i j}-\hat{R}_{i} \hat{R}_{j}\right) n^{2} k^{2} R^{2}\right] \mathrm{e}^{i n k R} \mathrm{e}^{-\kappa k R} .
\end{aligned}
$$

Here in the exponential factors, $n$ is the real part of the refractive index and $\kappa$ is the imaginary part, representing a coefficient of extinction. Combining the localized (2) and nonlocalized (5) contributions to the overall rate of SPDC thus gives

$$
\begin{aligned}
\sum_{A} M_{A}+\sum_{A, B} M_{A B} \\
=\sum_{A} \frac{i}{4}\left(\frac{\hbar \omega}{n}\right)^{\frac{3}{2}}\left(\frac{q}{\varepsilon_{0} V}\right)^{\frac{1}{2}} e_{i} \bar{e}_{k}^{\prime} \chi_{i(j k)}^{(2)}(-\omega,-\omega ; 2 \omega) \\
\quad \times\left[\bar{e}_{j}^{\prime}+\bar{e}_{m}^{\prime} \frac{\varepsilon_{0}}{n^{2} N} \chi_{l m}^{(1)}(-\omega, \omega) \sum_{B} e^{-i n k^{\prime} \cdot r_{B}} V_{j l}\left(n k^{\prime}, \boldsymbol{r}_{B}\right)\right] .
\end{aligned}
$$

Equations (1) and (8) demand a summation over all sites $A$. Since all sites in the material are constitutionally identical, the overall interactions at every site are similar; for each input photon the engagement with each annihilation site can be approximated as an independent process. The summation over $A$ therefore multiplies both terms of Eq. (8) by the same factor, related to the overall availability of interaction sites and competition between them for the role of $A$. For the following calculations we are only concerned with the relative contribution of the delocalized mechanism to the SPDC rate.

The significance of the emerging dependence on the linear susceptibility, evaluated at the wavelength of the down-converted light, should not be mistaken. It does not signify that the nonlocal mechanism simply involves scattering one in a pair of emergent photons. Such a process would not involve all of the time orderings of events that are necessarily accounted for in the sum leading to Eq. (5); moreover, the coherence in the output would be lost through the inelastic character of the process at each site. It should be appreciated that the true electrodynamic mechanism exploits a finite, rather than supposedly infinitesimal, extent of photon interaction.

In order to ascertain the relative significance of interacting $A-B$ pairs at varying distances, we next calculate the sum over all points $B$, subject to a constraint $\boldsymbol{r}_{B} \leq C$, where $C$ is an arbitrary distance from position $A$. The sum is 
evaluated over all sites $B$ for which the distance $\boldsymbol{r}_{B}$ is less than or equal to the chosen cutoff distance $C$; in effect $C$ is the radius of an imagined sphere centered on $A$, defining the region within which nonlocal interactions are to be included. The relevant part of (8) can then be written as a function of $C$,

$$
\sigma_{j l}(C) \equiv \sum_{B}^{r_{B} \leq C} e^{-i n k^{\prime} \cdot r_{B}} V_{j l}\left(n k, \boldsymbol{r}_{B}\right)
$$

It is instructive to model the process within a primitive cubic lattice. To this end point $A$ is defined as the origin; every surrounding position with integer values for the $x, y$, and $z$ coordinates (in units of the notional unit-cell length) is occupied by one optical center labeled $B$. For each $B$, the distance from $A$ is the magnitude of its position vector $\boldsymbol{r}_{B}$, given by the center's coordinates as $\boldsymbol{r}_{B}=\sqrt{ }\left(x^{2}+y^{2}+z^{2}\right)$.

By numerical simulation we calculate $\sigma_{z z}(C)$ for a range of discrete values of $C$ up to five output wavelengths, based on the placement of $A$ at the center of a $101^{3}$ point cubic lattice. The wave vector $\boldsymbol{k}^{\prime}$ is chosen to be off alignment with the lattice, and to have a wavelength $\lambda=2 \pi c / \omega$ equal to ten times the model unit-cell length. As shown in Fig. 3, the sum described by Eq. (9) converges after a distance of around $3.5 \lambda$ towards the true value of $\sigma_{z z}$, a global sum over all $B$ that in this case has magnitude $\varepsilon_{0}\left|\sigma_{z z}\right|=15300 \lambda^{-3}$. In addition to progression towards an asymptote, there is an oscillatory component: the inset of Fig. 3, which displays the derivative of the $\sigma_{z z}$ curve with respect to $C$, highlights the effect of both exponential factors that appear at the end of Eq. (7). The oscillations in particular manifest the occurrence of both constructive and destructive interferences, according to the number of sites in each direction about $A$ as the delimiting sphere increases in capacity.

When the SPDC rate Eq. (1) is solved using the total matrix element given by (8), the two terms in the square

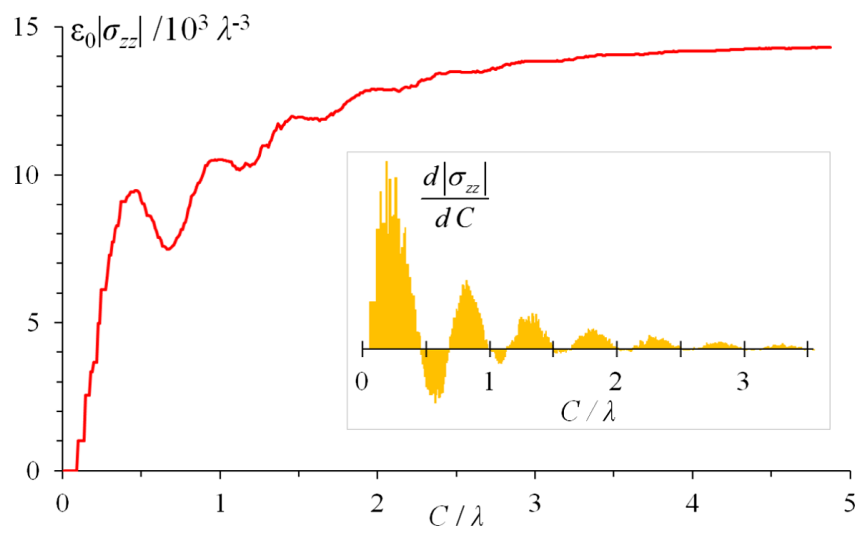

FIG. 3. Plot of the nonlocalized contribution to SPDC from the phase- and distance-weighted factor given by Eq. (9), normalized against $\varepsilon_{0}$, as a function of the cutoff distance $C$. The inset shows the derivative of the main graph. brackets of (8) give the relative contributions of singlecenter SPDC and the delocalized mechanism. In simplified form, taking the scalar part of all tensors, the rate Eq. (1) becomes

$$
\begin{aligned}
\Gamma_{\mathrm{SPDC}} \propto & \left|1+\frac{\varepsilon_{0}}{n^{2} N} \chi^{(1)}(-\omega, \omega) \sigma\right|^{2} \\
= & 1+2 \operatorname{Re}\left(\frac{\varepsilon_{0}}{n^{2} N} \chi^{(1)}(-\omega, \omega) \sigma\right) \\
& +\left|\frac{\varepsilon_{0}}{n^{2} N} \chi^{(1)}(-\omega, \omega) \sigma\right|^{2} .
\end{aligned}
$$

The first rate term, here normalized to 1 , signifies the rate of the single-center down-conversion process; the other terms are attributable to the delocalized mechanism. The relative effect on SPDC of the nonlocal process can be estimated by solving (10) with the relevant values of $n, \sigma$, and the ratio $\chi^{(1)} / N$ for the material in question.

It is worth noting that the three terms in the expanded form of Eq. (10), emerging from time-dependent perturbation theory, in general diminish successively in magnitude. The progression in powers of the linear susceptibility (6) offers one means to mitigate the associated reduction in significance, by judicious exploitation of preresonance enhancement. The term involving the square of the linear susceptibility would nonetheless remain smallest in magnitude.

To secure an indicative figure, relevant to a typical pair-generation material, we take the example of BBO $(\beta$ barium borate), for which the inverse of the true unit-cell volume $N=5 \times 10^{26} \mathrm{~m}^{-3}$. If the pump beam has wavelength $266 \mathrm{~nm}$, and a phase-matching direction is chosen such that $n=1.7$, then we can employ the approximation $\chi^{(1)}=n^{2}-1$ to determine the linear susceptibility [26]. The magnitude of the scalar $\sigma$ can be estimated from our calculation of a representative component of the generic $\sigma$ tensor, as $\sigma=3\left|\sigma_{z z}\right|$. In this case, the result of Eq. (10) is that approximately eight correlated pairs of photons are produced from delocalized SPDC, per ten thousand pairs attributable to the single-center SPDC mechanism. In other words, photon pairs are occasionally produced in which each photon emerges from a different point in space.

The result has a number of aspects with interesting significance. First, it signifies that the assumption of a common origin for each pair of photons emitted in SPDC is an approximation. In fact, a direct corollary of the mechanism we have described is the possibility that two downconverted photons that are indeed created at identical (or immeasurably close) points might indeed originate from the conversion of two different pump photons (considering point $B$ in Fig. 1 to be site $A$ for another such process). Such a possibility erodes the fidelity of state entanglement in the down-converted photons. Secondly, nonlocal downconversion must to some extent degrade the quality of images in applications such as ghost imaging [27], based on the separation of correlated photon twins: a physical 
mechanism emerges for a lack of fidelity that might otherwise have been more loosely attributed to noise. This is an issue of considerable significance in the context of factors more widely limiting optical resolution [28]. And thirdly, the form of dependence on linear susceptibility does indicate that optically dense materials exhibit a greater propensity for nonlocal down-conversion; as noted earlier, exploiting preresonance enhancement of SPDC in the wings of an optical absorption band disproportionately favors more delocalized emission. These are all features that deserve closer attention where they impinge on quantum optics and imaging applications.

We are pleased to acknowledge valuable assistance in the computational work from our colleague Dr. Garth A. Jones. Financial support in the form of a University of East Anglia (UEA) research studentship to K. A.F. is gratefully recorded.

[1] R. W. Boyd, Nonlinear Optics (Academic Press, New York, 2003).

[2] D. L. Andrews and P. Allcock, Optical Harmonics in Molecular Systems (Wiley-VCH, Weinheim, 2002).

[3] M. Müller, S. Bounouar, K. D. Jöns, M. Glässl, and P. Michler, Nat. Photonics 8, 224 (2014).

[4] S. P. Walborn, C. Monken, S. Pádua, and P. S. Ribeiro, Phys. Rep. 495, 87 (2010).

[5] P. G. Kwiat, K. Mattle, H. Weinfurter, A. Zeilinger, A. V. Sergienko, and Y. Shih, Phys. Rev. Lett. 75, 4337 (1995).

[6] A. S. Solntsev, A. A. Sukhorukov, D. N. Neshev, and Y. S. Kivshar, Phys. Rev. Lett. 108, 023601 (2012).

[7] H. Jin, F. M. Liu, P. Xu, J. L. Xia, M. L. Zhong, Y. Yuan, J. W. Zhou, Y. X. Gong, W. Wang, and S. N. Zhu, Phys. Rev. Lett. 113, 103601 (2014).

[8] J. W. Silverstone et al., Nat. Photonics 8, 104 (2014).

[9] M. McLaren, T. Mhlanga, M. J. Padgett, F. S. Roux, and A. Forbes, Nat. Commun. 5, 3248 (2014).
[10] J. L. O’Brien, G. J. Pryde, A. G. White, T. C. Ralph, and D. Branning, Nature (London) 426, 264 (2003).

[11] T. B.Pittman, Y. H. Shih, D. V. Strekalov, and A. V. Sergienko, Phys. Rev. A 52, R3429 (1995).

[12] M. P. Edgar, D. S. Tasca, F. Izdebski, R. E. Warburton, J. Leach, M. Agnew, G. S. Buller, R. W. Boyd, and M. J. Padgett, Nat. Commun. 3, 984 (2012).

[13] F. J. Duarte, Quantum Optics for Engineers (CRC Press, Boca Raton, 2014).

[14] L. Shi, E. J. Galvez, and R. R. Alfano, Sci. Rep. 6, 37714 (2016).

[15] R. Horodecki, P. Horodecki, M. Horodecki, and K. Horodecki, Rev. Mod. Phys. 81, 865 (2009).

[16] E. A. Power, Introductory Quantum Electrodynamics (American Elsevier Publishing Co., New York, 1965).

[17] W. Healy, Nonrelativistic Quantum Electrodynamics (Academic Press Inc. London, 1982).

[18] D. P. Craig and T. Thirunamachandran, Molecular Quantum Electrodynamics: An Introduction to Radiation-Molecule Interactions (Dover Publications, Mineola, NY, 1998).

[19] R. Woolley, Adv. Quantum Chem. 32, 167 (1998).

[20] E. A. Power and T. Thirunamachandran, Am. J. Phys. 46, 370 (1978).

[21] D. L. Andrews and D. S. Bradshaw, Eur. J. Phys. 25, 845 (2004).

[22] G. Juzeliūnas, Phys. Rev. A 53, 3543 (1996).

[23] L. Mandel and E. Wolf, Optical Coherence and Quantum Optics (Cambridge University Press, Cambridge, 1995), p. 871.

[24] R. D. Jenkins, D. L. Andrews, and L. C. D. Romero, J. Phys. B 35, 445 (2002).

[25] A. Salam, Molecular Quantum Electrodynamics. LongRange Intermolecular Interactions (Wiley, Hoboken, NJ, 2010).

[26] J. Lin, M.-H. Lee, Z.-P. Liu, C. Chen, and C. J. Pickard, Phys. Rev. B 60, 13380 (1999).

[27] R. S. Aspden, D. S. Tasca, R. W. Boyd, and M. J. Padgett, New J. Phys. 15, 073032 (2013).

[28] M. Paúr, B. Stoklasa, Z. Hradil, L. L. Sánchez-Soto, and J. Rehacek, Optica 3, 1144 (2016). 